\title{
Study of Automobile Driving Axle Test Bench Power Based on Closed Power Flow Principles
}

\author{
Ji Junling ${ }^{1, a}$, Wang Qiang ${ }^{2, b}$ and Wang Wei, \\ 1,2,3 HeiLongjiang Institute of Technology, HarBin, China \\ a136576183@qq.com, b630702666@qq.com, ${ }^{\text {c7 } 746602542 @ q q . c o m ~}$
}

Keywords: Automobile driving axle; Test bench; closed power flow; Performance test

\begin{abstract}
The paper analyzes the layout structure and working principle of driving axle performance closed test bench. The design concepts of test bench drive mechanism and loading mechanism are described on the basis of confirming the design scheme. Layout reasonability of the design structure is analyzed, and the mechanical stiffness and strength therefore are checked. Therefore, the test bench can complete running-in test and gear wear test. Mechanical closed power flow principle is utilized for developing a set of drive mechanism in the device for testing driving axle mechanical efficiency, stiffness, fatigue strength and lubrication.
\end{abstract}

\section{Introduction}

Heavy automobile driving axle performance and life test belong to important items of bench test of heavy automobile drive, and have important positions in trunk test equipment. Because the development of heavy automobile driving axle assembly gear fatigue test system has important research value and practical significance. Foreign heavy vehicle manufacturer conducted more detailed life test study on the reliability, for example, U.S. BURKE Company, UK ROMAX Company and SMT Manufacturing Technology Co., Ltd. Germany RENK Company and SCHENCK Company as well as Austria AVL Company are provided with considerable strength and market in the aspect of automotive driving axle test. Auto part test technology has been rapidly developed in foreign countries in recent years with continuous development of sensor technology, electronic technology and computer technology. Foreign automobile driving axle manufacturer not only adopts test equipment in the stages of product development, product design and effect validation, but also heavily utilizes on-line testing equipment with advanced testing equipment in the production and manufacturing links, namely, on the production line, on the assembly line and in the unmanned workshop. Testing equipment, testing instrument and spare parts are arranged in the whole processing process. The layout not only can be used for accurately determining whether the product is qualified or not, but also can correctly judge the quality out-of-control state-cause more importantly by test data analysis and processing. Since the test requires that the true vehicle actual condition should be simulated as similarly as possible, and the measurement parameters and functions are excessive, dedicated test bench must be built for testing the performance and life[1].

Because closed power flow test bench can complete mechanical efficiency measurement, fatigue service, lubrication and other tests demanding longer duration only through selecting smaller electric motors (only mechanical loss efficiency consumed by the closed system is provided) under the condition of loading the system in advance. The test bench is characterized by less power consumption, low investment and low power consumption. The driving axle has high mechanical efficiency and high power loss; thereby the topic develops a device suitable for automobile driving axle performance test. Closed power flow principles are adopted as design principles to achieve the purposes of energy saving, easy manipulation and strong applicability[2].

\section{The study on the automobile driving axle test bench power}

The test bench with closed power flow is referred to as closed test bench. The test bench with unclosed power flow is referred to as open test bench. The closed test bench has prominent 
characteristic of saving energy, which is used for fatigue test with longer test period. Common closed test benches include the follows: mechanical -loading closed driving axle assembly gear fatigue test bench (widely used at home and abroad), hydraulic loading closed driving axle assembly gear fatigue test bench, electricity closed driving axle assembly gear fatigue test bench and the like. Open test bench is easy to realize automatic control with wider test range. It is more generally used in performance tests, such as NQ510 type driving axle test bench produced by U.S. Gleason Company. In addition, there are many other open test benches. Partial energy recovery can be conducted in order to save energy, and it is widely used in Europe, America and Japan .The generally adopted test instruments include torque and rotation speed sensors in the driving axle assembly gear fatigue test. In addition, secondary instrument supported in the test include torque and speed meter, power meter, efficient instrument and the like in recent years. They provide convenient condition for bench test, and are easy to achieve automatic operation and measurement. The layout positions of the power devices and the power flow direction have direct impact to the system power loss. Rational layout of power devices and confirmation of power flow direction can control the system loss power to the minimum. Closed automobile driving axle reliability test bench and the best power device arrangement schemes can greatly reduce test energy consumption and effectively save the cost of test[3-4].

\section{Test Principle and Power Device Layout Scheme of Closed Test Bench}

The structure of the closed automobile driving axle assembly reliability test bench is shown in Figure1. It forms a closed system by main speed reducer, auxiliary gear boxes and loading device. The closed force moment is loaded through the loading system. Meshing force is generated among all gears in the whole closed system. The power device outside the closed system is used for completing the operation of the whole system, and meanwhile, the power loss generated due to heating in the closed system can be compensated. At this time, the energy consumed by the power device only accounts for a small part in the system. It can not be rotated to the reverse direction by support. Then, the closed system is disconnected. The loading pinion gear is pushed to the loading gear wheel and fixed with tools. The loading small motor is turned on subsequently. After the torque is increased by speed reduction of the small gear box, the larger torque is shown in Fig.1.

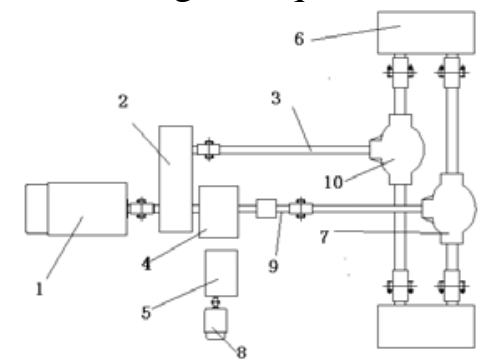

1- Running motor 2-Gear box A 3-Transmission shaft 4- Loading gear 5- Worm speed reducer 6- Gear box B 7 -Tested driving shaft 8- Loading motor 9- Torque sensor 10- Accompanying test driving shaft

Fig1. Closed Test Bench Principles

A motor is used for replacing the engine as driving force, and the driving gear box is driven to operate by the coupling in order to reduce the test bench structure, improve the controllability, reduce noise and pollution, and conserve energy. The driving gear box is further used for driving the loading chuck and loading gear wheel. Then, it is transferred to the tested driving axle assembly samples through the torque rotation speed sensor drive axle. Then, it passes through the gear boxes on both sides and the accompanying test driving axle assembly on the main test workpiece with the same model as the main test workpiece, and then is connected with the driving gear box through the transmission shaft, thereby forming a torque closed loop structure. Closed loading of the test bench refers that the loading motor drives the gear pair in the loading gear box and the worm pair drives the shiftable loading pinion gear. Loading process is as follows: the test bench motors are firstly turned off. Eight connecting bolts between the chuck behind the gear box and the loading gear wheel are loosened. Then, a special clamp is clamped in the slot outside the chuck. The clamp is transferred into 
the system behind the gear box through the meshing of the loading pinion gear and the loading gear wheel. The real time display torque value of the torque and rotation speed meter is observed. The loading is stopped when it reaches the target torque. The bolt can be used for connecting and fixing the chuck and the loading gear wheel under the condition. The special clamp can be removed; the loading pinion gear is exited, thereby preventing it from being meshed with the loading gear wheel. The internal torque loading in the system is completed then; the test bench can be turned on. Corresponding torque will be applied to the tested driving axle assembly and accompanying test driving axle assembly. The power flow flowing direction is shown in Fig2.

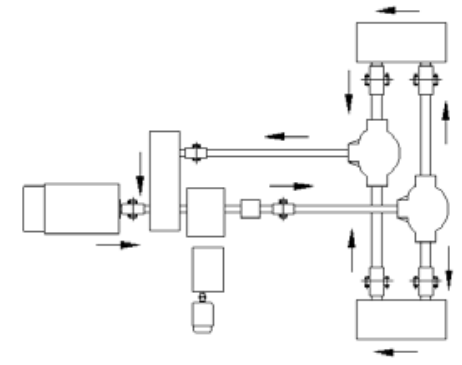

Fig2. Test Bench Power Flow Flowing Direction Schematic Diagram

\section{Transmission Mechanism and Loading Mechanism Design}

(1) Drive Motor Selection

The test bench selects technical parameters of a brand passenger car driving axle as benchmark. The parameters are as follows: The maximum power is $125 \mathrm{Kw} / 2300 \mathrm{rpm}$. The torque is $580 \mathrm{Nm} / 1300$ 1500rpm. The gear box gear 1 transmission ratio is 6.098. Gear R transmission ratio is 5.98. The driving axle main reduction ratio is 4.556 . The selected storage coefficient is $\mathrm{K}=1.5$ in order to meet wide application of the test bench. The selected drive motor model is Y200L1-2 according to 'Machine Design' manual. Its parameters are as follows: the rated power is $30 \mathrm{KW}$; and the full load speed is $2950 \mathrm{r} / \mathrm{min}$.

(2) Determination of Gear Box [5-6]

The transmission schematic diagram of gear box A is shown in Fig.3. The gear tooth number is $z_{1}=30, z_{2}=30 \times 1.5=45$, the gear $z_{3}$ and gear $z_{2}$ are the same, gear $z_{4}$ and gear $z_{1}$ are the same. The transmission schematic diagram of gear box B is shown in Fig.4. The gear tooth number is $z_{B 1}=30, z_{B 2}=30 \times 1.5=45$, the gear $z_{B 4}$ and the gear $z_{B 1}$, the gear $z_{B 2}$ and gear $z_{B 3}$ are the same two by two.
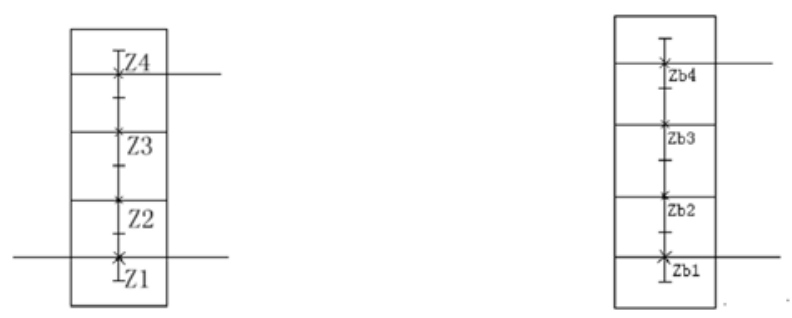

Fig3. Gear Box A Transmission Schematic Diagram (left)

Fig 4. Gear Box B Transmission Schematic Diagram (right)

(3) Power Calculation of Loading Small Motor[5-6]

The torque of loading small motor is transferred to the loading gear wheel through gear transmission, worm transmission and belt transmission. When the torque is transferred, the transmission energy is lost. We can calculate the loss according to the transfer efficiency and power demanded for loading. Concrete method is as follows: the loading speed should not be too high in order to timely and accurately observe the load torque value, $n_{q} \leq 6 \mathrm{r} / \mathrm{min}=18^{\circ} / \mathrm{s}$ can be selected, thereby the motor power value can be estimated according to the following formula: 


$$
P_{x d}=\frac{T_{q} \bullet n_{q}}{9550} \bullet \frac{1}{n_{j z}}
$$

In the formula, ${ }^{n_{j z}}-$ loading gear box transmission efficiency;

$$
\begin{aligned}
& n_{j z}=n_{\text {spurgear }} \times n_{\text {worm }} \times n_{\text {belt }} \times n_{\text {loadinggear }} \\
& =0.95 \times 0.75 \times 0.95 \times 0.97 \approx 0.66
\end{aligned}
$$

Therefore, it is calculated according to the maximum value

$$
P_{x a}=\frac{900 \times 6}{9550} \times \frac{1}{0.66}=0.80 \mathrm{KW},
$$

Y802-2, motor of $\mathrm{Y} 802-2, \mathrm{P}=1.1 \mathrm{KW}, \mathrm{n}=2825 \mathrm{rpm}$ is selected according to 'Mechanical Design’ manual.

(4) Worm and Turbine Design

Worm material is 45 steel. The tooth surface is hardened. The hardness is $\geq 45 \mathrm{HRC}$. Turbine material is ZCuAL10Fe3 with sand casting, $\mathrm{v}<2 \mathrm{~m} / \mathrm{s}$. $[\sigma]_{H}=160 \mathrm{MPa}$ is obtained according to the table. Worm head quantity $Z_{1}=1$. The turbine tooth quantity is $Z_{2}=Z_{1} i=5 \times 50=50$. The torque transmitted by the turbine is

$$
T_{2}=T_{1} i \eta=9.55 \times 10^{6} \times \frac{1.1 \times 2.5}{3000} \times 50 \times 0.75=328125 \mathrm{~N} \bullet \mathrm{mm} .
$$

Since the load is stable, the load factor is $\mathrm{K}=1.1 \mathrm{~m}=5 \mathrm{~mm}$. The diameter factor is $\mathrm{q}=10$. The pitch circle diameter is $d_{1}=50$. Turbine pitch circle diameter is $d_{2}=z_{2} m=50 \times 5=250 \mathrm{~mm}$. The worm lead angle is $r=\arctan \frac{z_{1}}{q}=\arctan \frac{1}{10}=5.71^{\circ}$. The center distance is $a=\frac{m}{2}\left(q+z_{2}\right)=150 \mathrm{~mm}$

\section{Summary}

Mechanical closed power flow principles are utilized to develop a set of transmission mechanism in the device for testing driving axle mechanical efficiency, stiffness, fatigue strength and lubrication. Because closed power flow test bench can complete mechanical efficiency measurement, fatigue service, lubrication and other tests demanding longer duration only through selecting smaller electric motors (only mechanical loss efficiency consumed by the closed system is provided) under the condition of loading the system in advance. The test bench is characterized by less power consumption, low investment and low power consumption. The driving axle has high mechanical efficiency and high power loss.

\section{Acknowledgement}

Project source: Heilongjiang Department of Science and Technology(GZ11A206)

\section{References}

[1] Huang Weigang, Wang Xuyong, Wang Xianzheng, etc. Research and development of automobile driving axle experimental device [J]. Shanghai Jiaotong University Journal, 1998, (12)

[2] $\mathrm{Hu}$ Shuihua, Pan Shirong, Wu Yongqiao, Mechanical part accumulated fatigue reliability calculation [J]. Wuhan: Journal of Wuhan Technology Institute,2004

[3] Wu Liyan, Wang Buying. Mechanical transmission system reliability analysis [J]. Beijing: Mechanical transmission, 2003

[4] Xu Hongping, Ying Fuqiang, Song Lingling. Design and research of mechanical transmission system multi-functional test bench [J] - Electrical and Mechanical Engineering ,2002 (03)

[5] QC / T 533.2000 Test methods of automobile driving axle [S], 2000

[6] QC / T 534.2000 Test evaluation indicator of automotive drive axle [S], 2000 